Pacific Journal of Mathematics

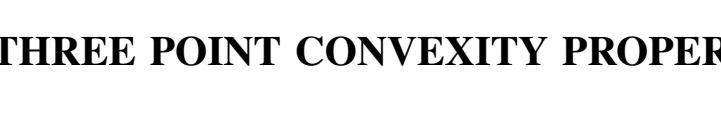




\title{
A THREE POINT CONVEXITY PROPERTY
}

\author{
F. A. VAlentine
}

There exist an interesting variety of set properties determined by placing restrictions on each triple of points of the set. It is the purpose here to study those closed sets in the $n$-dimensional Euclidean space $E_{n}$ (in particular the plane $E_{2}$ ) which satisfy the following condition.

Definition 1. A set $S$ in $E_{n}$ is said to possess the three-point convexity property $P_{3}$ if for each triple of points $x, y, z$ in $S$ at least one of the closed segments $x y, y z, x z$ is in $S$.

The principal result obtained in this paper appears in Theorem 2 . In order to achieve this result a series of lemmas and theorems is first established. Most of these are also of independent interest.

1. Closed connected sets in $E_{n}, n \geq 1$. In this section we assume that $S$ is a closed connected set in $E_{n}, n \geqq 1$. The concept of local convexity is a useful one for our purpose, so we restate the well-known definition.

DeFinition 2. A set $S$ is said to be locally convex at a point $q \in S$ if there exists an open sphere $N$ with center at $q$ such that $S \cdot N$ is convex. If a set is locally convex at each of its points, it is said to be locally convex.

Notation 1. The open segment determined by points $x$ and $y$ is denoted by $(x y)$, whereas $x y$ denotes the closed segment. The line determined by $x$ and $y$ is denoted by $L(x, y)$. The boundary of a set $S$ is $B(S)$, and $H(S)$ denotes the closed convex hull of $S$. The symbol + stands for set union, and the symbol - stands for set product.

THEOREM 1. Let $S$ be a closed connected set in $E_{n}(n \geqq 1)$ which has property $P_{3}$. Then either $S$ is convex or $S$ is starlike with respect to each of its points of local nonconvexity (It may be starlike elsewhere).

Proof. If $S$ is locally convex, then by a theorem of Tietze [4, pp. 697-707], [2, pp. 448-449], the set $S$ is convex, in which case it is starlike with respect to each of its points. Hence, suppose $S$ is not locally convex, and let $q \in S$ be a point of local nonconvexity. This implies that in each spherical neighborhood $N_{i}$ of $q$, there exist points

Received June 26, 1956. 
$x_{i}$ and $y_{i}$ of $S$ such that $\left(x_{i} y_{i}\right) \cdot S=0$ (see Notation 1). Choose any point $x \in S$. Property $P_{3}$ implies that either $x y_{i}$ or $x x_{i}$ is in $S$. If the radius of $N_{i}$ is $1 / i$, then as $i \rightarrow \infty$ the set $x x_{i}+x y_{i}$ converges to $q x$, which then must belong to $S$. This completes the proof.

REMARK 1. The set of all starlike points of a set $S$ is called the convex kernel of $S$. The convex kernel of a set $S \subset E_{n}$ is convex. See Brunn [1].

COROLlaRY 1. Each point of local nonconvexity of the set $S$ in Theorem 1 is contained in the boundary of the convex kernel of $S$.

Corollary 2. For the set $S$ above, let $H$ be any $r$-dimensional plane section of $S$, where $(1 \leqq r \leqq n-1)$. Then either $H \cdot S$ is starlike or $H \cdot S$ consists of two convex components.

Proof of Corollary 2. If $H \cdot S$ is connected, then since $H \cdot S$ has property $P_{3}$, Theorem 1 implies $H \cdot S$ is starlike. If $H \cdot S$ is not connected, property $P_{3}$ implies trivially that $H \cdot S$ consists of two and only two components, each of which must be convex.

COROLlaRY 3. Each component of the complement of $S$ is unbounded. This is an immediate consequence of the starlikeness of $S$.

2. Closed connected sets in $E_{2}$. We restrict ourselves to closed connected sets in $E_{2}$ in this section, and the following definitions are useful.

Definition 3. A component of the complement of a closed connected set $S$ is called a residual domain of. $S$. A cross-cut xy of a residual domain $K$ of $S$ is a closed segment such that $x \in S, y \in S$ and $(x y) \subset K$ (See Notation 1).

Definition 4. An isolated point of local nonconvexity of $S$ is called a $p$-point. A point of $S$ which is a $p$-point or a limit point of $p$-points is called a $q$-point.

Lemma 1. Each open segment (uv) of the convex kernel of $S$ contains no q-points of $S$. (see Corollary 1).

Proof. Suppose $w$ is a $q$-point contained in $(u v)$. Clearly $S \not \subset L(u, v)$ (see Notation 1). Choose $z \in S-L(u, v)$. Since $u v$ belongs to the convex kernel of $S$, we have triangle $u z v \subset S$. But this implies that each sufficiently small neighborhood of $w$ contains no cross-cuts of the com- 
plement of $S$, since such a cross cut $x y$ would have to have its interior $(x y)$ in one of the open half-planes bounded by $L(u, v)$.

Lemma 2. Let $S$ be a closed connected set in $E_{2}$ having property $P_{3}$. Then if $S$ is not convex, it contains at least one isolated point of local nonconvexity.

Proof. Let $x y$ be a cross-cut of a residual domain $K$ of $S$. Since $S$ is closed and connected, the set $K-(x y)$ is the union of two mutually exclusive open sets, denoted by $K_{1}$ and $K_{2}[3$, p. 118]. Since $S$ is starlike, Corollary 3 implies that one and only one of these two sets is bounded. Let it be $K_{1}$, and denote the boundary $B\left(K_{1}\right)-(x y) \equiv C\left(K_{1}\right)$. The set $B\left(K_{1}\right)$ is a continuum [3, p. 124]. Since $K_{1}$ is a bounded domain, and since $C\left(K_{1}\right) \cdot(x y)=0$, it follows that $C\left(K_{1}\right)$ is a continuum. Define $B_{1}$ to be the set of points $z_{1} \in C\left(K_{1}\right)$ such that $x z_{1} \subset S$, and define $B_{2}$ to be the points $z_{2} \in C\left(K_{1}\right)$ such that $y z_{2} \subset S$. Since $x y \not \subset S$, property $P_{3}$ implies $C\left(K_{1}\right)=B_{1}+B_{2}$. Moreover, $x \in B_{1}, y \in B_{2}$, and moreover $B_{1}$ and $B_{2}$ are each closed since $S$ and $C\left(K_{1}\right)$ are closed. Hence, since $C\left(K_{1}\right)$ is a continuum, it is well-known that $B_{1} \cdot B_{2} \neq 0$. Hence, let $p \in B_{1} \cdot B_{2}$, and we must have $x p \subset S, y p \subset S$, so that $K_{1}$ is interior to triangle $x p y$. Since $p \in B\left(K_{1}\right)$, it is clear that each neighborhood of $p$ contains a crosscut of $K_{1}$, so that $p$ is a point of local nonconvexity of $S$.

To prove that $p$ is an isolated point of local nonconvexity, observe that the lines $L(x, p)$ and $L(y, p)$ determine four $V$-shaped domains, each bounded by two rays. Order these $V_{1}, V_{2}, V_{3}, V_{4}$ so that $x y p \subset \bar{V}_{1}$, and so that the sets $V_{i}$ are arranged consecutively in a clockwise direction about the point $p$. Suppose a $p$-point $p_{1} \in \bar{V}_{1}-p$ exists. Then since $p_{1} x+p_{1} y \subset S$, we would have $K_{1} \subset x y p_{1}$, which would violate the fact $p \in B\left(K_{1}\right)$. Suppose a $p$-point, say $p_{1}$ exists in $V_{2}$. But this implies that $x p p_{1} \subset S, y p p_{1} \subset S$. But this again would violate the fact $p \in B\left(K_{1}\right)$. In exactly the same way $V_{4}$ contains no $p$-point of $S$. Now consider $V_{3}$. If $p_{1}$ is a $p$-point of $S$ in $V_{3}$, then $y p p_{1}+x p p_{1} \subset S$, which implies that $p$ is an isolated $p$-point since $V_{1}$ contains no $p$-point of $S$. Finally, Lemma 1 implies that no sequence of $p$-points of $S$ can exist on $L(x, p) \cdot \bar{V}_{3}$ or on $L(y, p) \cdot \bar{V}_{3}$ having $p$ as a limit point. Thus we have shown that $p$ is an isolated $p$-point of $S$.

REMARK 2. Let $x y$ be the cross-cut in the above proof, and let $p$ be the associated isolated $p$-point. Then the closed triangle $x y p$ is such that the set $x y p \cdot S$ is the union of two convex sets having only the point $p$ in common. One of these convex sets contains $x p$ and is denoted by $C(x p)$, and the other denoted by $C(y p)$ contains $x y$. 
Proof. Let $L_{i}$ and $L$ be lines parallel to $x y$, such that $L_{i}$ separates $p$ and $x y$, and such that $p \in L$. Let $H_{i}$ be the closed half-plane bounded by $L_{i}$ and containing $x y$. Suppose $L_{i} \rightarrow L$ as $i \rightarrow \infty$ so that $H_{i+1} \supset H_{i}$. Since $S \cdot H_{i} \cdot x y p$ is locally convex, by Tietze's Theorem [4, loc. cit.] each of its components is convex. Property $P_{3}$ implies that there are at most two such components. The fact that $x y \not \subset S$, implies there are exactly two such components. Denote them by $C_{i}$ and $D_{i}$. Clearly $C_{i+1} \supset C_{i}, D_{i+1} \supset D_{i}$, and hence $C_{i}$ and $D_{i}$ converge to convex sets having $p$ in common. They have only $p$ in common, otherwise $p$ would not be a boundary point of $K_{1}$ as defined in the proof of Lemma 2. One of the convex sets contains $x p$ and the other $y p$ so that the notation in the remark is justified.

Definition 5 . Let $Q$ be the set of $q$-points of $S$.

REMARK 3. Corollary 1 implies that $Q$ is contained in the boundary of its own convex hull $H(Q)$, designated by $B(H)$.

LEMma 3. The boundary of $H(Q)$ is connected, and it can contain at most one ray.

Proof. Since $H \equiv H(Q)$ is convex, if $B(H)$ were not connected, it would have to consist of two parallel lines (this is known). However, Lemma 1 would then imply that each of these parallel lines would contain at most two $q$-points. But this would imply that $Q$ is bounded in which case $B(H)$ would be connected. If $B(H)$ contained two rays, then Lemma 1 would again imply that $Q$ is bounded, which would again be contradictory.

Definition 6. An edge of the boundary $B(H)$ is a closed segment $x y$ or a closed ray $x \infty$ whose endpoints are $q$-points. An open half-plane whose boundary contains $x y($ or $x \infty)$, and which does not intersect $H(Q)$ is called an open half-plane of support, and it is denoted by $W$.

Lemma 4. Let $W$ be an open half-plane of support to $H(Q)$, which abuts on an edge $x y($ or $x \infty)$. Then $H(Q)+W \cdot S$ is a convex subset of $S$.

Proof. If $u \in H(Q)$ and if $v \in S \cdot W$, then $u v \subset S$, since $S$ is starlike with respect to $u$. This, together with the facts $x \in B(S), y \in B(S)$, and property $P_{3}$ imply that $u v \cdot x y \neq 0$ (or $u v \cdot x \infty \neq 0$ ), so that $u v \subset H(Q)+$ $W \cdot S$. Suppose $u \in S \cdot W, v \in S \cdot W$. Let $z \in(x y)$ or $(x \infty)$. If $:(u v) \not \subset S$, since $u z \subset S, v z \subset S$, then triangle $u v z$ would contain a $p$-point of $S$ (See the first paragraph of the proof of Lemma 2). But this is impossible, since $W$ contains no $p$-points of $S$, and since by Lemma 1 the open segment $(x y)$ or $(x \infty)$ contains no $p$-points of $S$. Hence $H(Q)+$ 
$W \cdot S$ is convex. It should be observed that if $H(Q) \equiv x y$, then $H(Q)+$ $W \cdot S$ may or may not be closed.

Lemma 5. Let $x_{i} y_{i}$ be a countable number of pairwise disjoint edges in $B(H) \equiv B(H(Q))$. Assume that $B(H)$ contains at least three edges, and let $W_{i}$ be the open half-plane of support to $H(Q)$ whose boundary contains $\left(x_{i} y_{i}\right)\left(x_{i} y_{i}\right.$ may be $\left.x_{i} \infty\right)$.

Then the set $H(Q)+S \cdot \Sigma_{i} W_{i}$ is a closed convex set.

Proof. Without loss of generality establish an order on the boundary $B(H)$, and assume that in terms of this order, $x_{i}$ is the beginning of the edge $x_{i} y_{i}$ and that $y_{i}$ is the endpoint of $x_{i} y_{i}$. Select any two disjoint edges $x_{i} y_{i}$ and $x_{j} y_{j}$, and without loss of generality assume that $x_{i}, y_{i}, x_{j}, y_{j}$ fall in an order so that an arc of $B(H)$ has $x_{i}$ and $y_{j}$ as its endpoints, and so that all four points lie on this arc in the order given above $(B(H)$ may be unbounded). Let the convex set which is bounded by the two lines $L\left(x_{i}, y_{j}\right)$ and $L\left(x_{j}, y_{i}\right)$, and which contains the quadrilateral $x_{i} y_{i} x_{j} y_{j}$ be denoted by $V$. The segments $x_{i} y_{i}$ and $x_{j} y_{j}$ divide $V$ into three parts; one is the closed quadrilateral $x_{i} y_{i} x_{j} y_{j}$; the second is a three sided closed polygonal set adjacent to $x_{i} y_{i}$ and denoted by $\Delta\left(x_{i}, y_{i}\right)$; the third is a three sided closed polygonal set adjacent to $x_{j} y_{j}$ and denoted by $\Delta\left(x_{j}, y_{j}\right)$. The last two sets may or may not be bounded. If the edge $x_{j} y_{j}$ is a ray $x_{j} \infty$ instead, then the same type of division occurs, in which $L\left(x_{i}, \infty\right)$ is a line parallel to the ray $x_{j} \infty$ so that $J\left(x_{j}, \infty\right)$ has two bounding sides instead of three. We must have $S \cdot W_{i} \subset \Delta\left(x_{i}, y_{i}\right)$, for if this were not so, it is easily seen that either $x_{i}$ or $y_{i}$ would be an interior point of a triangle which would belong to $S$. But this would contradict the fact that $x_{i} \in B(S), y_{i} \in B(S)$. Similarly $S \cdot W_{j} \subset \Delta\left(x_{j}, y_{j}\right)$. This is true whether $x_{j} y_{j}$ is a finite segment or a ray $x_{j} \infty$.

Now, choose two points $u$ and $v$ in $U \equiv H(Q)+S \cdot \Sigma_{i} W_{i}$. If $u$ and $v$ are in $H(Q)+S \cdot W_{i}$, then Lemma 4 implies $u v \subset U$. If $u \in S \cdot W_{i}$ and $v \in S \cdot W_{j}$, then by the preceding paragraph $u \in \Delta\left(x_{i}, y_{i}\right), v \in \Delta\left(x_{j}, y_{j}\right)$ (or $\left.v \in \Delta\left(x_{j}, \infty\right)\right)$. Since $V \equiv \Delta\left(x_{i}, y_{i}\right)+x_{i} y_{i} x_{j} y_{j}+\Delta\left(x_{j}, y_{j}\right)$ is convex, and since $\Delta\left(x_{i}, y_{i}\right) \cdot x_{i} y_{i} x_{j} y_{j}=x_{i} y_{i}$, we have $u v \cdot x_{i} y_{i} \neq 0$, whence $u v \subset U$.

To prove that $U$ is closed, observe first that if there are a finite number of disjoint sets $x_{i} y_{i}$ (there are at least three edges) then $U$ is closed, since $\bar{W}_{i} \cdot S \subset \Delta\left(x_{i}, y_{i}\right)$ implies $\overline{W_{i} \cdot S} \subset W_{i} \cdot S+B(H)$. If there are an infinite number of sets $W_{i}$, then let $s$ be a limit point of an infinite sequence of sets $W_{i_{n}} \cdot S$. Since $W_{i_{n}} \subset \Delta\left(x_{i_{n}}, y_{i_{n}}\right)$ by fixing $\left(x_{j}, y_{j}\right)$ of the preceding paragraph, it follows that $\left(x_{i_{i n}}, y_{i_{n}}\right) \rightarrow q$, a fixed point of $B(H)$, as $i_{n} \leftarrow \infty$. However, since in this situation, we must have $\Delta\left(x_{i_{n}}, y_{i_{n}}\right) \rightarrow q$ as $i_{n} \rightarrow \infty$, it follows that $s=q \in H(Q)$. Hence, it is clear that $U$ is 
closed, since $H(Q)$ is closed.

THEorem 2. Suppose $S$ is a closed connected set in $E_{2}$ such that for each triple of points $x, y, z$ in $S$ at least one of the segments $x y, y z, x z$ is in $S$.

Then $S$ is expressible as the union of three or fewer closed convex sets having a nonempty intersection. The number three is best.

Definition 7. Let $N$ denote the cardinality of the set of $p$-points of $S$ in Theorem 2.

THEOREM 3. If $N$ is not an odd integer greater than 1, then $S$ can be expressed as the union of two or fewer closed convex sets having a nonempty intersection.

Proofs of Theorems 2 and 3 . We recall that $Q$ is the closure of the set of $x$-points of $S$. The proof is divided into 5 cases, depending upon the value of $N$. The five cases are: $N=1 ; N=2 ; N=2 m>1 ; N=2 m$ $+1>1 ; N=\infty$.

Case 1. $N=1$. Let $Q=p$, and let $C$ be a circle with center at $p$ and having radius $r$. The set $S \cdot C$ is a closed connected set having property $P_{3}$ and having $p$ as its only $p$-point. If $S \cdot C$ satisfies the conclusions of either Theorem 2 or Theorem 3 , it is quite clear that $S=$ $\lim S \cdot C$ as $r \rightarrow \infty$ will satisfy the same conclusions. Let the boundary of the convex hull $H(S \cdot C)$ be $D(H)$, since $B(H)$ stands for the boundary of $H(Q)$. The rest of the proof will show incidentally that $D(H) \cdot S$ has one, two or four components.

Suppose $D(H) \cdot S$ has exactly three components and designate these by $B_{i}(i=1,2,3)$. It is easy to show that $B_{i} \neq\{p\}(i=1,2,3)$. Choose points $x_{i} \in B_{i}$ with $x_{i} \neq p(i=1,2,3)$. Property $P_{3}$ implies that at least one of the intervals $x_{1} x_{2}, x_{2} x_{3}, x_{3} x_{1}$ is in $S$. Suppose $x_{1} x_{2} \subset S$. Since $x_{i} \in D(H) \quad(i=1,2)$, and since $B_{1} \cdot B_{2}=0$, we must have $L\left(x_{1}, x_{2}\right) \cdot S=x_{1} x_{2}$. If $p \notin\left(x_{1} x_{2}\right)$, then let $H_{12}$ be the closed half-plane bounded by $L\left(x_{1}, x_{2}\right)$ and not containing $p$. Since $x_{1} \in B_{1}, x_{2} \in B_{2}$ with $B_{1} \cdot B_{2}=0$, there must exist a cross cut of the complement of $S$ in $H_{12}$. However, by the proof of Lemma 2, there would exist a $p$-point in $H_{12} \cdot S$ which contradicts the fact that $Q=p$. Hence $p \in\left(x_{1} x_{2}\right)$. Since $p \in\left(x_{1} x_{2}\right)$, if $x_{1} x_{3} \subset S$, the proof of Lemma 2 would again imply the existence of a $p$-point in that closed half-space bounded by $L\left(x_{1}, x_{3}\right)$ which does not contain $p$. However this contradicts $Q=p$. Hence, $x_{1} x_{3} \not \subset S$. Similarly $x_{2} x_{3} \not \subset S$. Property $P_{3}$ and the closure of $S$ implies that for points $x \in B_{1}$ sufficiently near $x_{1}$, we have $x x_{2} \subset S, \quad x x_{3} \not \subset S, \quad x_{2} x_{3} \not \subset S$. Applying the same reasoning to $x, x_{2}, x_{3}$ that we applied to $x_{1}, x_{2}, x_{3}$, we get $p \in\left(x x_{2}\right)$ for all $x$ near 
$x_{1}$. This can only be true if $B_{1}=x_{1}$. Similarly, $B_{2}=x_{2}$. However, since $B_{3}$ is contained in only one of the open half planes bounded by $L\left(x_{1}, x_{2}\right)$, the facts $B_{i}=x_{i}(i=1,2)$ simply that $x_{1} x_{2} \subset D(H) \cdot S$, which contradicts the fact $B_{1} \cdot B_{2}=0$. Hence $D(H) \cdot S$ cannot have exactly three components. Suppose $D(H) \cdot S$ has at least four components, and designate four of these by $B_{i}(i=1,2,3,4)$. The above argument implies that $B_{i}=x_{i}$ $\in D(H) \cdot S$, and these can be renumbered so that $p \in\left(x_{1} x_{2}\right), p \in\left(x_{3} x_{4}\right)$. Clearly any fifth component $B_{5}$ could not exist, since the above argument applied to $x_{1}, x_{2}, x_{3}$ and $x_{5} \in B_{5}$ would yield $p \in\left(x_{1} x_{2}\right), \quad p \in\left(x_{3} x_{5}\right)$, so that $x_{5}=x_{4}$, a contradiction. Thus if $D(H) \cdot S$ has more than two components, then $S \cdot C$ is the union of two line segments having an interior point in common.

Now, suppose $D(H) \cdot S$ has exactly two components denoted by $B_{1}$ and $B_{2}$. Let the end points of $B_{i}$ be $x_{i}$ and $y_{i}$ ordered so that $y_{1} x_{2}$ and $y_{2} x_{1}$ are cross-cuts of the complement of $S$. The points $x_{i}$ and $y_{i}$ need not be distinct. We will prove that each of the sets $P_{i} \equiv H\left(B_{i}+p\right)+$ $C\left(x_{i} p\right)+C\left(y_{i} p\right),(i=1,2)$ is convex. (See Remark 2 following Lemma 2 for the definitions of $C\left(x_{i} p\right)$ and $\left.C\left(y_{i} p\right)\right)$. Property $P_{3}$ and the fact that $D(H) \cdot S=B_{1}+B_{2}$ implies that $B_{i}+x_{i} p+y_{i} p$ is the boundary of $H\left(B_{i}+p\right)$. We will prove that $P_{1}$ is convex. Since each of the sets $H\left(B_{1}+p\right)$, $C\left(x_{1} p\right), C\left(y_{1} p\right)$ is convex, to show that $P_{1}$ is convex, it suffices to select points $z \in H\left(B_{1}+p\right), u \in C\left(x_{1} p\right), v \in C\left(y_{1} p\right)$, and to show that $u v+u z+v z$ $\subset P_{1}$. We must have $u v \cdot x_{1} p \neq 0, u v \cdot y_{1} p \neq 0$, for if this were not so, the fact $D(H) \cdot S=B_{1}+B_{2}$ would imply that $u v \cdot P_{2} \neq 0$. However, this would contradict property $P_{3}$. Hence, we have $u v \subset x_{1} y_{1} p+C\left(x_{1} p\right)+C\left(y_{1} p\right)$. Thus $u v \subset P_{1}$. In the same manner $u z \subset P_{1}, v z \subset P_{1}$, so that $P_{1}$ is convex. The same argument applies to $P_{2}$.

Finally if $D(H) \cdot S$ has exactly one component, and $Q=p$, it can be shown readily that there exists a line through $p$ which divides $S \cdot C$ into two closed convex sets having $p$ in common. This completes the proof for $N=1$, and oddly enough it appears to be the most difficult to prove.

Case 2. $\quad N=2$. Let $Q=p_{1}+p_{2}$. The line $L\left(p_{1}, p_{2}\right)$ divides the plane into two open half-planes $W_{i}(i=1,2)$. Lemma 4 implies that $W_{i} \cdot S$ is convex. If $W_{1} \cdot S=0$, then $S=\bar{W}_{2} \cdot S+S \cdot L\left(p_{1}, p_{2}\right)$ yields the desired conclusions of Theorem 2 and 3 . Hence suppose $W_{i} \cdot S \neq 0 \quad(i=1,2)$. Let $U \equiv \overline{W_{1} \cdot S}+\overline{W_{2} \cdot S}$. If $U$ is convex, then $S \equiv U+S \cdot L\left(p_{1}, p_{2}\right)$ yields the desired decomposition. Suppose $U$ is not convex, then we can show that $S \cdot L\left(p_{1}, p_{2}\right)=U \cdot L\left(p_{1}, p_{2}\right)$, for suppose a point $u \in S \cdot L\left(p_{1}, p_{2}\right)-$ $U \cdot L\left(p_{1}, p_{2}\right)$ exists. Since $U$ is not convex, there exist points $x_{i} \in W_{i} \cdot S$ such that $x_{1} x_{2} \not \subset S$. Moreover $u x_{i} \not \subset S$, since $u \notin \overline{W_{i} \cdot S}$. However, this violates property $P_{3}$. Thus if $U$ is not convex, $S=\overline{W_{1} \cdot S}+\overline{W_{2} \cdot S}$, and this is a desired decomposition of $S$ into two convex sets. 
Case 3. $N=2 m>2$. In this case the hull $H(Q)$ is a convex polygon, each segment of which is an edge having $p$-points as endpoints (See definition 6). Order the edges $x_{i} x_{i+1}$ of the boundary $B(H)$ counterclockwise so that $\left(i=1,2, \cdots, 2 m ; x_{1}=x_{2 m+1}\right)$. The open half-plane of support to $H(Q)$ adjacent to $x_{i} x_{i+1}$ is denoted by $W_{i}$. By Lemma 5 each of the sets

$$
S_{1} \equiv H(Q)+S \cdot \sum_{i=1}^{m} W_{2 i-1}
$$

$$
S_{2} \equiv H(Q)+S \cdot \sum_{i=1}^{m} W_{2 i}
$$

is a closed convex set. Moreover, since $S \subset H(Q)+\sum_{i=1}^{2 m} W_{i}$ we have $S_{1}+S_{2}=S$.

Case 4. $\quad N=2 m+1>1$. As in Case 3 , let $e_{i} \equiv x_{i} x_{i+1} \quad(i=1, \cdots$, $2 m+1 ; x_{1}=x_{2 m+2}$ ) denote the ordered edges of $B(H)$, and define $S_{1}$ and $S_{2}$ as in (1).

Let

$$
S_{3} \equiv H(Q)+S \cdot W_{2 m+1} \text {. }
$$

By Lemma 5, the sets $S_{1}, S_{2}$ and $S_{3}$ satisfy the conclusions of Theorem 2 .

Case 5. $N=\infty$. In order to prove this case, the following definition is helpful.

DEFINITION 8. A connected closed subset $I$ of the boundary $B(H)$ is called a polygonal element if the following conditions hold:

(a) It is the closure of the union of edges of $B(H)$ (see Definition 6).

(b) Its endpoints (one, two or none) are limit points of $p$-points of $S$.

(c) If $I=B(H)$, then $I$ contains at most one limit point of $p$-points. If $I \neq B(H)$, then only its endpoints (one or two) are limit points of p-points.

Observe that these conditions imply that a polygonal element is maximal in the sense that it is not a proper subset of a larger polygonal element.

The number of polygonal elements of $B(H)$ is countable, hence we can well-order them easily. Let $I_{1}, I_{2}, \cdots, I_{n} \cdots$ designate such a wellordering.

For each polygonal element $I_{n}$, divide the edges it contains (see Definition 6) into two classes $M_{n}^{1}$ and $M_{n}^{2}$ such that no two edges of $M_{n}^{i}(i=1,2)$ are adjacent, that is, have an endpoint in common. It may 
happen that one of the $M_{n}^{i}$ may be empty. For each edge $e \in M_{n}^{i}$ we let $W_{e}^{i}$ denote the open half-plane of support to $B(H)$ whose boundary contains $e$. Define

$$
F_{n}^{i}=\sum_{e \in M_{n}^{i}} W_{e}^{i} \cdot S \quad(i=1,2),
$$

and let

$$
S_{i}=H(Q)+\sum_{n} F_{n}^{i} \quad(i=1,2) .
$$

Since each edge in $M_{n}^{i}$ is separated from each edge in $M_{m}^{i}(n \neq m)$, Lemma 5 implies that $S_{1}$ and $S_{2}$ are closed convex subsets of $S$. Moreover, since for each point $x \in S$, either $x \in H(Q)$, or $x$ is contained in some $W_{e}^{i} \cdot S$, we have $S=S_{1}+S_{2}$ and $S_{1} \cdot S_{2} \neq 0$.

To prove that the number "three" in Theorem 2 is best consider the familiar two cell formed by a five-pointed star. It is a simple matter to verify that this set has property $P_{3}$, and that it cannot be expressed as the union of two convex sets. The analogous $2 m+1$ pointed star behaves the same way.

\section{Concluding remarks.}

(a) It should be noted that the converse of Theorem 2 is not true. For instance, the set consisting of three segments $x x_{i}(i=1,2,3)$, where each angle $\angle x_{i} x x_{j}=120^{\circ}(i \neq j)$, is the union of three convex sets; yet it does not have property $P_{3}$.

(b) It would be of interest to characterize those sets in $E_{2}$ which are the union of two closed convex sets. It appears that such a characterization will follow from an investigation of the cardinality of the set $B(K) \cdot B(S)$, where $K$ is the convex kernel of $S$.

(c) The theory in $E_{3}$ needs to be settled. In view of $\S 1$, it is natural to ask the question. What are the closed connected sets in $E_{3}$ such that each of its plane sections is either starlike or the union of two disjoint convex sets?

\section{REFERENCES}

1. H. Brunn, ̈̈ber Kerneigebiete, Math. Ann. 73 (1913), 436-440.

2. V. Klee, Convex sets in linear spaces, Duke Math. J., 18, No. 2 (1951), 443-466.

3. M. H. A. Newman, Topology of plane sets of points, second edition (reprinted 1954), Cambridge University Press.

4. H. Tietze, Über Konvexheit im kleinen und im grossen und über gewisse den Punkten einer Menge zugeordnete Dimensionszahlen, Math. Z. 28 (1929), 697-707. 



\section{PACIFIC JOURNAL OF MATHEMATICS}

\section{EDITORS}

H. L. ROYDEN

Stanford University

Stanford, California

R. A. Beaumont

University of Washington

Seattle 5, Washington

\section{A. L. Whiteman}

University of Southern California

Los Angeles 7, California

E. G. Straus

University of California

Los Angeles 24, California

\section{ASSOCIATE EDITORS}
E. F. BECKENBACH
C. E. BURGESS
M. HALL
E. HEWITT

\author{
A. HORN \\ V. GANAPATHY IYER \\ R. D. JAMES \\ M. S. KNEBELMAN
}

L. NACHBIN

I. NIVEN

G. SZEKERES

T. G. OSTROM

M. M. SCHIFFER
F. WOLF

K. YOSIDA

\section{SUPPORTING INSTITUTIONS}

\author{
UNIVERSITY OF BRITISH COLUMBIA \\ CALIFORNIA INSTITUTE OF TECHNOLOGY \\ UNIVERSITY OF CALIFORNIA \\ MONTANA STATE UNIVERSITY \\ UNIVERSITY OF NEVADA \\ OREGON STATE COLLEGE \\ UNIVERSITY OF OREGON \\ UNIVERSITY OF SOUTHERN CALIFORNIA
}

\author{
STANFORD UNIVERSITY \\ UNIVERSITY OF UTAH \\ WASHINGTON STATE COLLEGE \\ UNIVERSITY OF WASHINGTON \\ AMERICAN MATHEMATICAL SOCIETY \\ CALIFORNIA RESEARCH CORPORATION \\ HUGHES AIRCRAFT COMPANY \\ THE RAMO-WOOLDRIDGE CORPORATION
}

Mathematical papers intended for publication in the Pacific Journal of Mathematics should be typewritten (double spaced), and the author should keep a complete copy. Manuscripts may be sent to any of the editors. All other communications to the editors should be addressed to the managing editor, E. G. Straus at the University of California, Los Angeles 24, California.

50 reprints per author of each article are furnished free of charge; additional copies may be obtained at cost in multiples of 50 .

The Pacific Journal, of Mathematics is published quarterly, in March, June, September, and December. The price per volume (4 numbers) is $\$ 12.00$; single issues, $\$ 3.50$. Back numbers are available. Special price to individual faculty members of supporting institutions and to individual members of the American Mathematical Society: $\$ 4.00$ per volume; single issues, $\$ 1.25$.

Subscriptions, orders for back numbers, and changes of address should be sent to Pacific Journal of Mathematics, 2120 Oxford Street, Berkeley 4, California.

Printed at Kokusai Bunken Insatsusha (International Academic Printing Co., Ltd.), No. 10, 1-chome, Fujimi-cho, Chiyoda-ku, Tokyo, Japan.

PUBLISHED BY PACIFIC JOURNAL OF MATHEMATICS, A NON-PROFIT CORPORATION

The Supporting Institutions listed above contribute to the cost of publication of this Journal, but they are not owners or publishers and have no responsibility for its content or policies. 


\section{Pacific Journal of Mathematics}

\section{Vol. 7, No. 2 \\ February, 1957}

William F. Donoghue, Jr., The lattice of invariant subspaces of a completely continuous quasi-nilpotent transformation ................... 1031

Michael (Mihály) Fekete and J. L. Walsh, Asymptotic behavior of restricted extremal polynomials and of their zeros.................... 1037

Shaul Foguel, Biorthogonal systems in Banach spaces ............... 1065

David Gale, A theorem on flows in networks ................... 1073

Ioan M. James, On spaces with a multiplication .................. 1083

Richard Vincent Kadison and Isadore Manual Singer, Three test problems in operator theory .................................... 1101

Maurice Kennedy, A convergence theorem for a certain class of Markoff processes........................................ 1107

G. Kurepa, On a new reciprocity, distribution and duality law ........ 1125

Richard Kenneth Lashof, Lie algebras of locally compact groups ........ 1145

Calvin T. Long, Note on normal numbers .................... 1163

M. Mikolás, On certain sums generating the Dedekind sums and their reciprocity laws ..................................... 1167

Barrett O'Neill, Induced homology homomorphisms for set-valued maps......................................... 1179

Mary Ellen Rudin, A topological characterization of sets of real numbers........................................... 1185

M. Schiffer, The Fredholm eigen values of plane domains 1187

F. A. Valentine, A three point convexity property .........

Alexander Doniphan Wallace, The center of a compact lattice is totally

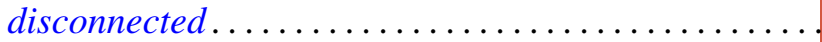

Alexander Doniphan Wallace, Two theorems on topological lattices.

G. T. Whyburn, Dimension and non-density preservation of mappings...

John Hunter Williamson, On the functional representation of certain algebraic systems ... 